\title{
Accretion Detection via Scanning Acoustic Microscopy in Microelectronic Components - Considering Symmetry Breaking Effects
}

\author{
Eva Grünwald ${ }^{1}$, René Hammer ${ }^{1}$, Jördis $\operatorname{Rosc}^{1}$, Bernhard Sartory ${ }^{1}$ and Roland Brunner ${ }^{1}$ \\ ${ }^{1 .}$ Materials Center Leoben Forschung GmbH, Leoben, Austria.
}

Currently, micro - and nano-electronic devices are pushed towards higher performance, increased functionality and higher integration density. A promising technique to achieve „More than Moore devices“ is 3D integration. Here, layers of active electronic components are connected vertically via socalled through silicon vias (TSVs). These TSVs are practically cylindrical holes with diameters in the range from $5-200 \mu \mathrm{m}$ in silicon which are filled or coated with conducting materials. The processing, design and the performance of TSVs faces many challenges and especially the defect detection, failure characterization and reliability analysis are in the focus of state of the art reliability studies $[1,2]$.

In this work, we consider scanning acoustic microscopy (SAM) as a tool of choice in the characterization of TSVs. With frequencies ranging up to the $\mathrm{GHz}$ regime, SAM provides sufficient lateral resolution and with fast sampling rates of state of the art ADC boards down to ps, SAM provides high axial resolution in the analysis of microelectronic samples. The non-destructive nature of the SAM analysis allows for subsequent analyses with other methods and minimizes the possibility of introducing new failure modes by the analysis itself. Moreover, SAM shows high potential with respect to rapid learning towards rapid in-line inspection of 3D integrated samples. Recently, the high potential for the automatization of SAM measurements concerning defects in tungsten coated TSVs was shown [2].

In the work reported here, the SAM analysis of metal coated TSVs of approximately $100 \mu$ m diameter was carried out. The analyzed sample was an array of TSV test structures, where different kinds of failure modes were artificially introduced in order to test the ability of SAM concerning their detection.

In the SAM measurement, an acoustic objective with a center frequency of $100 \mathrm{MHz}$ and an opening angle of the lens with about $60^{\circ}$ was used. The SAM analysis showed high sensitivity to accretions. Figure 1 (a) shows a SAM C-scan image of a TSV labeled with "TSV 1", where an irregularity in the TSV can be observed on the left hand side. In Figure 1 (b), the X-ray computed tomography (XCT) result for "TSV 1" is shown. The XCT shows only an indication of a possible failure. Therefore, no reliable measurement can be performed in Figure 1 (b). The SEM analysis of "TSV 1" shown in Figure 1 (c) shows an accretion and allows an estimation of the extension. The accretion shows an extension of approximately 30 times 10 microns parallel to the coating but only a very small extension reaching towards the center of the TSV.

In order to interpret the SAM results, elastodynamic finite integration (EFIT) simulations [3] of sound wave propagation in TSVs in two space dimensions were carried out, see Figure 2. The time-steps in the simulation are numbered from 1 to 5. The sound wave starts in time-step 1 from the right hand side of the TSV and propagates along the wall of the TSV. Figure 2 (a) shows a sound wave propagating on the wall of an intact TSV, whereas Figure 2 (b) shows the case of a sound wave propagating on the wall of a TSV with a small accretion. The accretion is marked red in time-step 1 in Figure 2 (b). From Figure 2, it can be seen, that the sound field in the symmetric intact TSV, Figure 2 (a) is disturbed by the presence 
of the accretion Figure 2 (b), especially in time-step 3 directly at the accretion. In the later time-step 5, the effect of the accretion on the wall is visible in the middle of the TSV.

Here, the sensitivity of SAM to accretions in metal coated TSVs was demonstrated, Figure 1 (a). The results were corroborated by high resolution X-ray computed tomography (Figure 1 (b)) and via SEM (Figure 1 (c)). In order to interpret the SAM results, EFIT simulations were carried out. From the simulations, it seems that a combination of the longitudinal sound waves, the Rayleigh waves and other wave modes in the TSVs can increase the sensitivity of SAM concerning the detection of accretions [4]. References:

[1] E. Grünwald et al, Microelectron. Reliab. 64 (2016), p. 370.

[2] S. Brand, et al, Microelectron. Reliab. 50 (2012), p. 1469.

[3] P. Fellinger, et al, Wave Motion 21 (1995), p. 47.

[4] The ams AG is acknowledged for their support. This work has received funding from ENIAC Joint undertaking under FP7 research (No.:621270) and FFG (No.:843740) and partly from the EU, H2020 (No.: 688225). Financial support by the Austrian Federal Government (in particular from

Bundesministerium für Verkehr, Innovation und Technologie and Bundesministerium für Wissenschaft, Forschung und Wirtschaft) represented by Österreichische Forschungsförderungsgesellschaft $\mathrm{mbH}$ and the Styrian and the Tyrolean Provincial Government, represented by Steirische

Wirtschaftsförderungsgesellschaft $\mathrm{mbH}$ and Standortagentur Tirol, within the framework of the COMET Funding Programme (No. 837900) is also gratefully acknowledged.
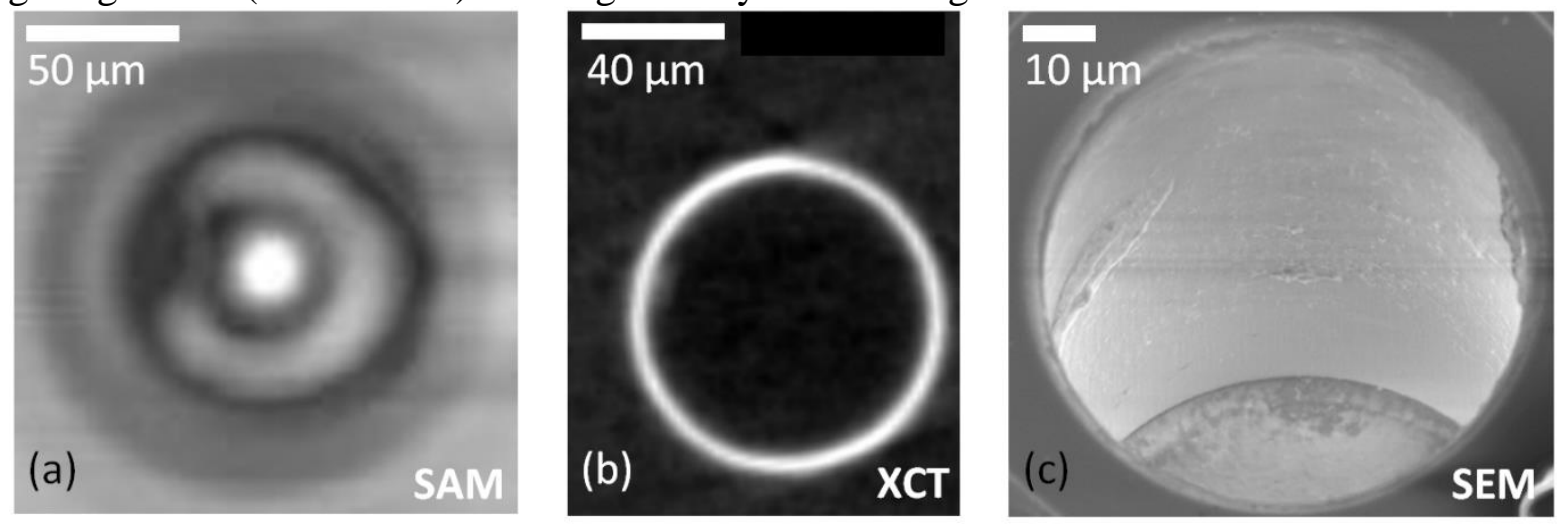

Figure 1. Accretion in "TSV 1", (a) SAM C-Scan, (b), XCT result and (c) SEM image.

(a)
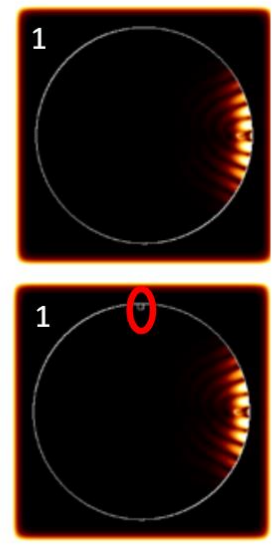
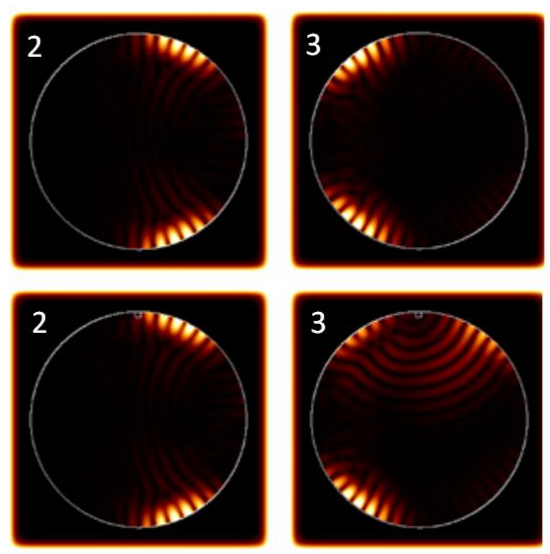

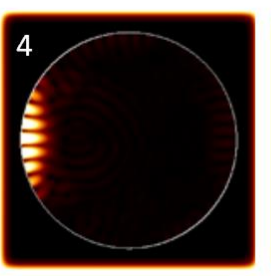

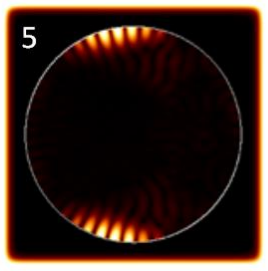
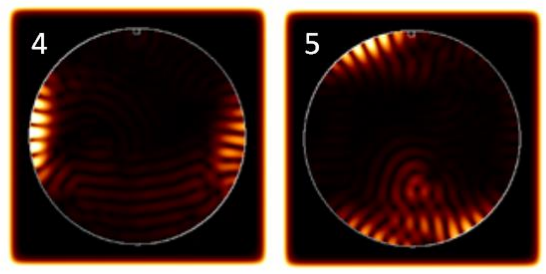

Figure 2. EFIT simulation of (a) sound wave propagation in intact TSV and (b) sound wave propagation in TSV with accretion. 